\title{
A pilot data analysis of a metabolomic HPLC-MS/MS study of patients with COPD
}

\author{
Barbora Novotna ${ }^{1-3, A-E}$, Mohammed Abdel-Hamidid,C,E,F, Vladimir Koblizek1,2,B,E,F, Michal Svoboda ${ }^{5, C, F}$, \\ Karel Hejduk ${ }^{5, C, E, F}$, Vit Rehacek ${ }^{6, B, E, F}$, Josef Bis ${ }^{7, B, E, F}$, Frantisek Salajka ${ }^{1,2, E, F}$ \\ ${ }^{1}$ Faculty of Medicine, Charles University in Prague, Hradec Králové, Czech Republic \\ 2 Department of Pneumology, University Hospital, Hradec Králové, Czech Republic \\ ${ }^{3}$ Department of Pneumology and Thoracic Surgery, Municipal Hospital Bulovka, Prague, Czech Republic \\ ${ }^{4}$ Department of Pharmaceutical Chemistry, Faculty of Pharmacy, Kuwait University, Kuwait \\ ${ }^{5}$ Institute of Biostatistics and Analyses, Faculty of Medicine, Masaryk University, Brno, Czech Republic \\ ${ }^{6}$ Transfusion Department, University Hospital, Hradec Králové, Czech Republic \\ ${ }^{7}$ Department of Cardioangiology, University Hospital, Hradec Králové, Czech Republic \\ A - research concept and design; $\mathrm{B}$ - collection and/or assembly of data; $\mathrm{C}$ - data analysis and interpretation; \\ $D$ - writing the article; $E$ - critical revision of the article; $F$ - final approval of the article
}

Address for correspondence

Barbora Novotna

E-mail: barbnovotna@gmail.com

Funding sources

The Czech team was supported by the program MZČR RVO (FNHK,00179906), PRVOUK P37/08 and I-LFHK.

Conflict of interest

None declared

Received on April 12, 2016

Reviewed on September 24, 2016

Accepted on February 2, 2017

DOI

$10.17219 /$ acem/68763

\section{Copyright}

Copyright by Author(s)

This is an article distributed under the terms of the

Creative Commons Attribution Non-Commercial License

(http://creativecommons.org/licenses/by-nc-nd/4.0/)

\section{Abstract}

Background. Chronic obstructive pulmonary disease (COPD) is a heterogeneous condition with multiple clinical faces. Metabolomic profiling studies small molecules present in biological samples by combined use of chromatography with mass spectrometry.

Objectives. The goal of our work was to perform a high performance liquid chromatography combined with tandem mass spectrometry (HPLC-MS/MS) metabolomic study to compare the concentrations of metabolites in COPD patients and in controls.

Material and methods. Participants were recruited at the University Hospital, Hradec Králové, Czech Republic, with the approval of the ethics committee. The analysis of blood samples was performed at Health Sciences (enter (HSC) in Kuwait. The blood samples were analyzed for concentrations of acylcarnitines and amino acids by high performance liquid chromatography (Waters 2690 HPLC; Waters, Milford, USA) and a triple-quadruple tandem mass spectrometer (Quattro LC, Micromass, Manchester, United Kingdom).

Results. Groups of 10 subjects with COPD and 10 healthy controls were analyzed. Carnitine analysis showed that the free carnitine to acylcarnitine ratio (CO/AC ratio) was significantly lower in COPD $(0.58 \mu \mathrm{M} / \mathrm{L}) \mathrm{com}$ pared to the controls $(0.73 \mu \mathrm{M} / \mathrm{L} ; \mathrm{p}=0.002)$. The mean $\mathrm{C} 8 / \mathrm{C} 2$ ratio in the COPD group was significantly higher $(0.03 \mu \mathrm{M} / \mathrm{L})$ - in the control group it was $0 \mu \mathrm{M} / \mathrm{L}(p=0.03)$. Amino acid analysis showed lower levels of phenylalanine in the COPD group $(22.05 \mu \mathrm{M} / \mathrm{L})$ compared to the controls $(30.05 \mu \mathrm{M} / \mathrm{L} ; p=0.008)$. The alanine concentrations were significantly lower in the COPD group $(173 \mu \mathrm{M} / \mathrm{L})$ than in the control group $(253 \mu \mathrm{M} / \mathrm{L} ; p=0.001)$. The pyroglutamate levels were higher in COPD $(1.58 \mu \mathrm{M} / \mathrm{L})$ than in the controls $(1 \mu \mathrm{M} / \mathrm{L} ; \mathrm{p}=0.040)$.

Conclusions. The carnitine and acylcarnitine levels in COPD subjects in this study possibly indicate a predisposition to atherosclerosis as a result of inadequate $\beta$-oxidation of fatty acids and show the presence of oxidative stress. Furthermore, the high sensitivity to changes in circulating amino acid levels may allow us to detect subclinical malnutrition and take early preventative interventions such as nutritional supplementation and patient education.

Key words: amino acids, chronic obstructive pulmonary disease, liquid chromatography-tandem mass spectrometry, carnitine, metabolomics 


\section{Introduction}

Chronic obstructive pulmonary disease (COPD) is a term referring to a heterogeneous group of chronic lung diseases marked by restricted airflow. Lung damage in COPD is nonreversible and progressive, but may be halted through proper treatment. The two most prevalent conditions characteristic for COPD are emphysema and chronic bronchitis, but new, not yet fully elucidated phenotypes such as asthma-COPD overlap syndrome (ACOS), pulmonary cachexia and frequent exacerbators are emerging. ${ }^{1-3}$ COPD has a severe impact on patients' health and quality of life. ${ }^{4,5}$

Metabolomic studies represent a modern trend in biological and clinical scientific work. ${ }^{6}$ Metabolomic profiling studies small molecules (molecular weight $<900 \mathrm{Da}$ ) present in biological samples. Originally, mainly nuclear magnetic resonance (NMR) was applied for this purpose. Presently, the combination of chromatographic methods with mass spectrometry (combinations of gas or liquid chromatography coupled to mass spectrometry - GC-MS or LC-MS) has become a widely applied analytical method for global profiling of metabolites. ${ }^{7}$

Only a limited number of metabolomic profiling studies was performed in patients with COPD. A limited number of papers are devoted to analyzing concentrated samples of exhaled air in patients with COPD using chromatographic methods or NMR spectroscopy. ${ }^{8-11}$

A metabolomic study based on the use of NMR spectroscopy was able to pinpoint the differences between serum of healthy individuals and COPD patients; particularly, decreased concentrations of lipoproteins and amino acids and increased concentrations of glycerolphosphocholine, acetate, ketone bodies, carnosine, $\mathrm{m}$-hydroxyphenylacetate, phenylacetyglycine, pyruvate, and $\alpha$-ketoglutarate. ${ }^{12}$ Another NMR metabolomic study demonstrated the method by investigating plasma and urine samples from 197 COPD patients and 195 adults without COPD. ${ }^{13}$ The findings were not conclusive and experiments need to be repeated and validated. On the other hand, the authors found this approach suitable for the identification of novel biomarkers, useful for determining COPD therapeutic outcomes. ${ }^{13}$ A metabolomic approach for studying COPD patients was applied to a defined cohort from the Evaluation of COPD Longitudinally to Identify Predictive Surrogate End-points (ECLIPSE) study. ${ }^{14}$ Serum from healthy subjects and Global Initiative for Chronic Obstructive Lung Disease (GOLD) stage II, III and IV patients with COPD was investigated using NMR spectrometry. The findings were confirmed by liquid chromatography with tandem mass spectrometry (LC-MS/MS) and correlated with other data. NMR spectrometry helped in identifying decreased concentrations of lipoproteins and N,N-dimethylglycine. On the other hand, increased concentrations of glutamine, phenylalanine, 3-methylhistidine, and ketone bodies were shown in GOLD stage IV COPD patients who also had decreased branched-chain amino acids (BCAAs) levels. There were found negative correlations between BCAAs, their degradation products, 3-methylhistidine, ketone bodies, and triglycerides on one side and cachexia on the other side. These concentrations positively correlated with systemic inflammation. ${ }^{14}$

In the ECLIPSE study, the concentrations of amino acids and dipeptides were measured by LC-MS/MS in groups of patients divided according to the GOLD characteristics. Differences in the levels of several amino acids and other small molecules were confirmed in the groups of COPD patients. ${ }^{15}$ The study showed differences among various groups of COPD patients (emphysema and no emphysema; cachectic and non-cachectic). Additionally, COPD patients were distinguished from 'healthy' smokers used as a control group. These results are in agreement with the findings of another study that concentrated on the differentiation of subjects with or without emphysema. ${ }^{16}$

High performance liquid chromatography combined with tandem mass spectrometry (HPLC-MS/MS) may be used for the identification of subtle differences in biomarkers between COPD patients, healthy controls, smokers and nonsmokers, etc. This method proved to be useful not only in metabolomic studies, but also in various proteomic investigations identifying proteins that could help differentiate healthy subjects from diseased groups of patients. ${ }^{17-19}$ Some proteins present at very low concentrations show different expression in various COPD patients, reflecting the possible pathogenic mechanisms and the extent and severity of lung remodeling. This has a potential in COPD diagnosis and disease activity tracking by biomarkers for the benefit of patients by improving the disease management.

The goal of our work was to perform a HPLC-MS/MS metabolomic study to compare the abundance of several small molecules in COPD patients and healthy volunteers.

\section{Material and methods}

\section{Diagnostic criteria for chronic obstructive pulmonary disease}

Chronic obstructive pulmonary disease was diagnosed on the basis of an evaluation of patients' lung functions, symptoms and history of exacerbations. All patients in this study performed a spirometry test and met the COPD diagnostic criteria of post-bronchodilator values of forced expiratory volume in $1 \mathrm{~s} /$ forced vital capacity $(\mathrm{FEV} 1 / \mathrm{FVC})<0.70 .^{1,2}$

\section{Patients}

Blood samples from COPD patients for this study were obtained from the participants registered in the Czech Multicenter Research Database of Chronic Obstructive Pulmonary Disease (ClinicalTrials.gov identifier NCT01923051). Blood samples from patients with coronary artery disease and healthy controls were obtained 
from the Department of Cardioangiology and the Transfusion Department of the University Hospital in Hradec Králové (Czech Republic), respectively. The ethics committee of the hospital approved the study. All participants signed an informed consent form. The analysis of blood samples was performed at the Department of Pharmaceutical Chemistry, Faculty of Pharmacy, Kuwait University (Kuwait).

The inclusion criteria for this study were: non-smokers or ex-smokers of at least 6 months, patients without acute exposition to carbon monoxide (CO) due to smoke or pollution inhalation (measured by smokerlyzer) and COPD patients with post-bronchodilator values of $\mathrm{FEV}_{1}<60 \%$.

Patients were excluded upon the following criteria: current smoker or ex-smokers of less than 6 months (CO levels $>10$ ppm), a known metabolic disease including diabetes type I and II, kidney disease with moderate to severe creatinine clearance, and the presence of coronary artery disease.

Venous blood was collected by standard venipuncture into BD Vacutainer ${ }^{\circledR}$ Heparin Tubes (Becton Dickinson, Mississauga, Canada). Heparinized blood was spotted onto a filter paper cards and left to dry at room temperature for at least $24 \mathrm{~h}$.

We analyzed 2 groups of subjects (Table 1). They included 10 subjects with COPD and 10 healthy controls. The mean age of the COPD group was 61.5 years with a male to female ratio ( $\mathrm{m}: \mathrm{f})$ of 1 . The mean age of the control group was 55 years with $\mathrm{m}$ :f of 1 . Although the healthy control group was younger, this difference was not significant $(\mathrm{p}=0.052)$. The mean body mass index (BMI) was matched in the COPD group $\left(25.3 \mathrm{~kg} / \mathrm{m}^{2}\right)$ and in the control group $\left(27.10 \mathrm{~kg} / \mathrm{m}^{2}\right)$, with $\mathrm{p}=0.496$. The COPD patients had an average fat free mass index (FFMI) of $18.79 \mathrm{~kg} / \mathrm{m}^{2}$; $\mathrm{FEV}_{1}$ of $33 \%$; and $\mathrm{FEV}_{1} / \mathrm{FVC}$ of 0.52 . Spirometric data and FFMI were not available for the control group.

\section{HPLC-MS/MS instrumentation}

The blood samples were analyzed for acylcarnitines and amino acids by a triple-quadruple tandem mass spectrometer (Quattro LC, Micromass, Manchester, UK) with a positive electrospray ionization probe. High performance liquid chromatography (Waters 2690 HPLC) with an autosampler (Waters, Milford, USA) was used for automatic injection. A designed tandem mass spectrometry (MS/ MS) program for automatic profiling of acylcarnitines and amino acids was used. The NeoLynx program (Quattro LC, Micromass, Manchester, United Kingdom) was used to determine the concentration of the diagnostic metabolites.

\section{HPLC-MS/MS method}

Using a BSD 400 Puncher (BSD Biosample punchers, Brendale, Australia), 2-3-mm disks were punched from the center of dried blood spots. The disks were placed in a capped glass for extraction of the blood with $200 \mu \mathrm{L}$
Table 1. Descriptive statistics

\begin{tabular}{|c|c|c|c|}
\hline & $\operatorname{COPD}(n=10)$ & Control $(n=10)$ & $p$-value \\
\hline Sex (male), n (\%) & $5(50.0 \%)$ & $5(50.0 \%)$ & 0.999 \\
\hline Age [years] & $61.50(28.00 ; 70.00)$ & $55.00(53.00 ; 61.00)$ & 0.052 \\
\hline BMI $\left[\mathrm{kg} / \mathrm{m}^{2}\right]$ & $25.30(19.47 ; 32.95)$ & $27.10(24.40 ; 29.40)$ & 0.496 \\
\hline FFMI $\left[\mathrm{kg} / \mathrm{m}^{2}\right]$ & $18.79(14.96 ; 25.79)$ & - & - \\
\hline $\mathrm{FEV}_{1}[\%]$ & $33.00(26.00 ; 56.00)$ & - & - \\
\hline $\mathrm{FEV}_{1} / \mathrm{FVC}$ & $0.52(0.30 ; 0.74)$ & - & - \\
\hline $\mathrm{CO}$ & $24.75(15.40 ; 44.20)$ & $33.40(19.10 ; 47.60)$ & 0.140 \\
\hline $\mathrm{C} 2$ & $12.45(8.10 ; 35.30)$ & $10.95(6.20 ; 14.10)$ & 0.076 \\
\hline $\mathrm{C} 3$ & $0.43(0.20 ; 0.67)$ & $0.26(0.00 ; 0.61)$ & 0.140 \\
\hline C4 & $0.00(0.00 ; 0.22)$ & $0.00(0.00 ; 0.19)$ & 0.351 \\
\hline C5 & $0.00(0.00 ; 0.22)$ & $0.04(0.00 ; 0.23)$ & 0.525 \\
\hline $\mathrm{C} 5-\mathrm{OH}$ & $0.04(0.00 ; 0.39)$ & $0.02(0.00 ; 0.36)$ & 0.716 \\
\hline $\mathrm{C} 8$ & $0.07(0.00 ; 1.34)$ & $0.00(0.00 ; 0.16)$ & 0.264 \\
\hline $\mathrm{C} 14$ & $0.00(0.00 ; 0.09)$ & $0.00(0.00 ; 0.10)$ & 0.564 \\
\hline C14:1 & $0.00(0.00 ; 0.18)$ & $0.00(0.00 ; 0.31)$ & 0.551 \\
\hline $\mathrm{C} 16$ & $0.56(0.00 ; 5.56)$ & $0.16(0.00 ; 2.85)$ & 0.412 \\
\hline $\mathrm{C} 16-\mathrm{OH}$ & $0.00(0.00 ; 0.04)$ & $0.00(0.00 ; 0.12)$ & 0.842 \\
\hline C18:1 & $0.42(0.00 ; 0.61)$ & $0.00(0.00 ; 0.74)$ & 0.090 \\
\hline AC total & $37.67(26.99 ; 81.04)$ & $45.44(26.90 ; 61.48)$ & 0.450 \\
\hline CO/AC & $0.58(0.53 ; 0.68)$ & $0.73(0.57 ; 0.86)$ & 0.002 \\
\hline $\mathrm{C} 3 / \mathrm{C} 16$ & $0.22(0.00 ; 0.61)$ & 0.07 (0.00; 0.84) & 0.589 \\
\hline $\mathrm{C} 3 / \mathrm{C} 2$ & $0.10(0.00 ; 0.16)$ & $0.22(0.00 ; 0.82)$ & 0.074 \\
\hline $\mathrm{C} 5 / \mathrm{C} 2$ & $0.00(0.00 ; 0.06)$ & $0.01(0.00 ; 0.09)$ & 0.648 \\
\hline $\mathrm{C} 8 / \mathrm{C} 2$ & $0.03(0.00 ; 0.50)$ & $0.00(0.00 ; 0.06)$ & 0.030 \\
\hline C14:1/C16 & $0.01(0.00 ; 0.44)$ & $0.00(0.00 ; 0.34)$ & 0.183 \\
\hline $\mathrm{C} 16-\mathrm{OH} / \mathrm{C} 16$ & $0.00(0.00 ; 0.06)$ & $0.00(0.00 ; 0.20)$ & 0.829 \\
\hline Glutaryl & $0.00(0.00 ; 0.36)$ & $0.00(0.00 ; 0.29)$ & 0.402 \\
\hline Valine & $85.95(57.20 ; 166.00)$ & $97.40(2.80 ; 134.00)$ & 0.910 \\
\hline Pyg & $1.58(0.00 ; 3.43)$ & $1.00(0.00 ; 1.96)$ & 0.040 \\
\hline Met & $0.00(0.00 ; 41.30)$ & $0.00(0.00 ; 0.00)$ & 0.317 \\
\hline Phe & $22.05(17.20 ; 34.20)$ & $30.05(21.10 ; 43.70)$ & 0.008 \\
\hline Tyr & $21.75(0.80 ; 171.00)$ & $27.85(22.20 ; 50.00)$ & 0.130 \\
\hline Cit1 & $13.20(0.00 ; 25.70)$ & $12.40(0.00 ; 26.40)$ & 0.940 \\
\hline Glyc & $90.40(77.20 ; 163.00)$ & $104.00(67.00 ; 146.00)$ & 0.496 \\
\hline Alanine & $173.00(141.00 ; 215.00)$ & $253.00(173.00 ; 346.00)$ & 0.001 \\
\hline Leu/Ile & $58.85(37.30 ; 69.40)$ & $64.65(51.40 ; 96.00)$ & 0.104 \\
\hline Phe/Tyr & $0.00(0.00 ; 3.43)$ & $0.00(0.00 ; 2.69)$ & 0.487 \\
\hline
\end{tabular}

BMI - body mass index; FFMI - fat free mass index; FEV 1 - forced expiratory volume in $1 \mathrm{~s}$; FVC - forced vital capacity; CO - free or nonacylated carnitine; C2-C18 - carnitine acylated with an acyl containing the indicated number of carbon atoms; - $\mathrm{OH}$ - a hydroxyl group on an acyl; AC - acylcarnitines; Pyg - pyroglutamic acid; Met - methionine; Phe - phenylalanine; Tyr - tyrosine; Cit - citrulline; Glyc - glycine; Leu - leucine; lle - isoleucine.

The concentrations of metabolites are in units $\mu \mathrm{M} / \mathrm{L}$; categorical variable is described by absolute (relative) frequency and tested by Fisher's exact test; continuous variable is described by median ( $5^{\text {th }}$ and $95^{\text {th }}$ percentiles) and tested by the Mann-Whitney test. 
of methanol, containing known concentrations of a mixture of isotopically labeled internal standards of diagnostic acylcarnitines and amino acids. After 20 min of shaking, the solvent was gently evaporated, and the residue was mixed with $80 \mu \mathrm{L}$ of butanolic $\mathrm{HCl}$ and heated at $65^{\circ} \mathrm{C}$ for $15 \mathrm{~min}$ in a capped glass tube. The solvent was evaporated again and the residue was finally reconstituted in $80 \mu \mathrm{L}$ of the mobile phase acetonitrile/water (80/20 v/v). A $20 \mu \mathrm{L}$ aliquot of each sample was directly injected into the mobile phase flowing to the ionization probe of the tandem mass spectrometer at a flow rate of $0.1 \mathrm{~mL} / \mathrm{min}$. A Waters 2690 HPLC with an autosampler was used for automatic injection. The run cycle time for each sample was 2-3 min from injection to injection. A designed MS/MS program for automatic profiling of acylcarnitines and amino acids was used. The NeoLynx program for Neonatal Screening (Quattro LC, Micromass, Manchester, United Kingdom) was used for automatic detection of abnormal samples. The values obtained for COPD patients and patients with coronary heart disease were compared to those of healthy controls.

\section{Statistical methods}

Standard descriptive statistics was applied for the analysis: absolute and relative frequencies for categorical

Table 2. Influence of sex on the results

\begin{tabular}{|c|c|c|c|c|c|c|}
\hline \multirow{2}{*}{$\begin{array}{l}\text { Carnitines and } \\
\text { amino acids }\end{array}$} & \multicolumn{3}{|c|}{ COPD } & \multicolumn{3}{|c|}{ Control } \\
\hline & female $(n=5)$ & male $(n=5)$ & $p$-value & female $(n=5)$ & male $(n=5)$ & $\mathrm{p}$-value \\
\hline $\mathrm{CO}$ & $24.60(18.70 ; 44.20)$ & $24.90(15.40 ; 25.50)$ & 0.602 & $24.60(19.10 ; 35.70)$ & $42.70(20.10 ; 47.60)$ & 0.117 \\
\hline $\mathrm{C} 2$ & $12.10(9.70 ; 35.30)$ & $12.90(8.10 ; 19.50)$ & 0.917 & $11.00(7.69 ; 14.10)$ & $10.90(6.20 ; 12.70)$ & 0.754 \\
\hline $\mathrm{C} 3$ & $0.47(0.20 ; 0.67)$ & $0.41(0.34 ; 0.64)$ & 0.917 & $0.16(0.00 ; 0.61)$ & $0.36(0.00 ; 0.54)$ & 0.916 \\
\hline C4 & $0.00(0.00 ; 0.20)$ & $0.07(0.00 ; 0.22)$ & 0.290 & $0.00(0.00 ; 0.10)$ & $0.00(0.00 ; 0.19)$ & 0.881 \\
\hline$C 5$ & $0.00(0.00 ; 0.22)$ & $0.00(0.00 ; 0.20)$ & 0.999 & $0.03(0.00 ; 0.09)$ & $0.04(0.00 ; 0.23)$ & 0.832 \\
\hline $\mathrm{C} 5 \mathrm{OH} \mathrm{C} 5-\mathrm{OH}$ & $0.00(0.00 ; 0.18)$ & $0.07(0.00 ; 0.39)$ & 0.577 & $0.00(0.00 ; 0.04)$ & $0.11(0.00 ; 0.36)$ & 0.034 \\
\hline $\mathrm{C} 8$ & $0.06(0.00 ; 1.34)$ & $0.10(0.00 ; 0.51)$ & 0.746 & $0.00(0.00 ; 0.14)$ & $0.00(0.00 ; 0.16)$ & 0.368 \\
\hline C14 & $0.00(0.00 ; 0.09)$ & $0.00(0.00 ; 0.05)$ & 0.638 & $0.00(0.00 ; 0.10)$ & $0.00(0.00 ; 0.04)$ & 0.521 \\
\hline C14:1 & $0.00(0.00 ; 0.18)$ & $0.00(0.00 ; 0.01)$ & 0.881 & $0.00(0.00 ; 0.03)$ & $0.00(0.00 ; 0.31)$ & 0.368 \\
\hline C16 & $0.29(0.00 ; 1.56)$ & $0.89(0.00 ; 5.56)$ & 0.245 & $0.00(0.00 ; 0.31)$ & $0.81(0.00 ; 2.85)$ & 0.034 \\
\hline $\mathrm{C} 16-\mathrm{OH}$ & $0.00(0.00 ; 0.03)$ & $0.00(0.00 ; 0.04)$ & 0.366 & $0.00(0.00 ; 0.06)$ & $0.00(0.00 ; 0.12)$ & 0.881 \\
\hline C18:1 & $0.40(0.00 ; 0.52)$ & $0.44(0.00 ; 0.61)$ & 0.916 & $0.00(0.00 ; 0.40)$ & $0.00(0.00 ; 0.74)$ & 0.521 \\
\hline ACTotal & $36.53(33.42 ; 81.04)$ & $38.81(26.99 ; 46.62)$ & 0.917 & $35.01(26.90 ; 50.33)$ & $49.39(35.56 ; 61.48)$ & 0.076 \\
\hline CO/AC & $0.59(0.55 ; 0.68)$ & $0.57(0.53 ; 0.66)$ & 0.347 & $0.71(0.65 ; 0.74)$ & $0.77(0.57 ; 0.86)$ & 0.117 \\
\hline $\mathrm{C} 3 \mathrm{C} 16$ & $0.15(0.00 ; 0.61)$ & $0.28(0.00 ; 0.34)$ & 0.675 & $0.00(0.00 ; 0.65)$ & $0.23(0.00 ; 0.84)$ & 0.147 \\
\hline $\mathrm{C} 3 \mathrm{C} 2$ & $0.09(0.00 ; 0.15)$ & $0.11(0.00 ; 0.16)$ & 0.287 & $0.07(0.00 ; 0.67)$ & $0.25(0.21 ; 0.82)$ & 0.075 \\
\hline $\mathrm{C} 5 \mathrm{C} 2$ & $0.00(0.00 ; 0.06)$ & $0.00(0.00 ; 0.06)$ & 0.906 & $0.01(0.00 ; 0.05)$ & $0.00(0.00 ; 0.09)$ & 0.911 \\
\hline $\mathrm{C} 8 \mathrm{C} 2$ & $0.02(0.00 ; 0.50)$ & $0.04(0.00 ; 0.19)$ & 0.829 & $0.00(0.00 ; 0.00)$ & $0.00(0.00 ; 0.06)$ & 0.317 \\
\hline $\mathrm{C} 14: 1 / \mathrm{C} 16$ & $0.00(0.00 ; 0.44)$ & $0.01(0.00 ; 0.12)$ & 0.737 & $0.00(0.00 ; 0.34)$ & $0.00(0.00 ; 0.00)$ & 0.136 \\
\hline $\mathrm{C} 16-\mathrm{OH} / \mathrm{C} 16$ & $0.00(0.00 ; 0.00)$ & $0.00(0.00 ; 0.06)$ & 0.136 & $0.00(0.00 ; 0.20)$ & $0.00(0.00 ; 0.15)$ & 0.881 \\
\hline Glutaryl & $0.00(0.00 ; 0.36)$ & $0.00(0.00 ; 0.00)$ & 0.136 & $0.00(0.00 ; 0.14)$ & $0.03(0.00 ; 0.29)$ & 0.290 \\
\hline Valine & $67.40(57.20 ; 103.00)$ & $95.30(80.90 ; 166.00)$ & 0.076 & $80.70(2.80 ; 110.00)$ & $103.00(77.10 ; 134.00)$ & 0.117 \\
\hline Pyg & $1.23(0.00 ; 3.43)$ & $2.27(1.18 ; 2.50)$ & 0.251 & $1.18(0.00 ; 1.96)$ & $0.00(0.00 ; 1.40)$ & 0.281 \\
\hline Met & $0.00(0.00 ; 41.30)$ & $0.00(0.00 ; 0.00)$ & 0.317 & $0.00(0.00 ; 0.00)$ & $0.00(0.00 ; 0.00)$ & 0.999 \\
\hline Phe & $21.80(17.20 ; 34.20)$ & $24.00(19.70 ; 24.50)$ & 0.463 & $29.60(21.10 ; 36.20)$ & $30.50(23.10 ; 43.70)$ & 0.754 \\
\hline Tyr & $26.30(13.40 ; 171.00)$ & $19.50(0.80 ; 31.50)$ & 0.251 & $28.00(22.20 ; 50.00)$ & $27.80(22.20 ; 44.60)$ & 0.675 \\
\hline Cit1 & $8.61(0.00 ; 14.30)$ & $18.90(5.96 ; 25.70)$ & 0.172 & $12.60(0.00 ; 26.40)$ & $9.26(0.00 ; 20.40)$ & 0.530 \\
\hline Glyc & 88.40 (77.20; 163.00) & $92.40(78.30 ; 120.00)$ & 0.754 & $113.00(82.00 ; 146.00)$ & $101.00(67.00 ; 119.00)$ & 0.465 \\
\hline Alanine & 147.00 (141.00; 193.00) & $176.00(157.00 ; 215.00)$ & 0.251 & $276.00(197.00 ; 307.00)$ & 230.00 (173.00; 346.00) & 0.917 \\
\hline Leulle Leu/lle & $57.10(37.30 ; 65.50)$ & $59.50(51.40 ; 69.40)$ & 0.175 & $55.50(51.40 ; 91.60)$ & $77.50(62.40 ; 96.00)$ & 0.175 \\
\hline PheTyr Phe/Tyr & $1.09(0.00 ; 3.43)$ & $0.00(0.00 ; 0.00)$ & 0.054 & $0.00(0.00 ; 0.92)$ & $0.00(0.00 ; 2.69)$ & 0.881 \\
\hline
\end{tabular}

$\mathrm{CO}$ - free or non-acylated carnitine; C2-C18 - carnitine acylated with an acyl containing the indicated number of carbon atoms; -OH - a hydroxyl group on an acyl; AC - acylcarnitines; Pyg - pyroglutamic acid; Met - methionine; Phe - phenylalanine; Tyr - tyrosine; Cit - citrulline; Glyc - glycine; Leu - leucine; lle - isoleucine.

The concentrations of metabolites are in units $\mu \mathrm{M} / \mathrm{L}$; continuous variable is described by median (5 $5^{\text {th }}$ and $95^{\text {th }}$ percentiles) and tested by the Mann-Whitney test. 
variables, and median supplemented by $5^{\text {th }}-95^{\text {th }}$ percentile range for continuous variables. Statistical significance of differences was tested using Fisher's exact test for categorical variables and the Mann-Whitney test for continuous variables. Spearman's correlation coefficient was used for the analysis of the relationship between continuous variables. Statistical analysis was computed using SPSS 22 (IBM Corporation, New York, USA; 2013).

\section{Results}

In the analysis of the concentrations of free carnitine and acylcarnitines, small variations between the 2 groups were noted. Most notably, the free carnitine to acylcarnitine ratio (C0/AC ratio) was significantly lower in the COPD group compared to the control group - COPD $0.58 \mu \mathrm{M} / \mathrm{L}$ (0.53; 0.68) and control group $0.73 \mu \mathrm{M} / \mathrm{L}(0.57 ; 0.86)$ with $\mathrm{p}=0.002$. The mean $\mathrm{C} 8 / \mathrm{C} 2$ ratio in the COPD group was significantly higher, $0.03 \mu \mathrm{M} / \mathrm{L}(0 ; 0.50)$, whilst it was $0 \mu \mathrm{M} / \mathrm{L}$ in the control group $(\mathrm{p}=0.03)$.

The analysis of amino acids showed significantly lower levels of phenylalanine in the COPD group compared to the controls. The phenylalanine level in the COPD group was $22.05 \mu \mathrm{M} / \mathrm{L}(17.20 ; 34.20)$ and in the control group, it was $30.05 \mu \mathrm{M} / \mathrm{L}(21.10 ; 43.70)$ with $\mathrm{p}=0.008$; the alanine levels were significantly lower in the COPD group, $173 \mu \mathrm{M} / \mathrm{L}(141 ; 215)$, than in the control group $253 \mu \mathrm{M} / \mathrm{L}(173 ; 346) ; \mathrm{p}=0.001$. The pyroglutamate levels were significantly higher in COPD patients, $1.58 \mu \mathrm{M} / \mathrm{L}$ (0; 3.43), than in the control group $-1 \mu \mathrm{M} / \mathrm{L}(0 ; 1.96)$; $\mathrm{p}=0.040$.

Gender had no influence on the metabolomic profile of COPD patients, and only a small effect in the control group (Table 2). In the control group, males had significantly higher levels of $\mathrm{C} 5 \mathrm{OH}(0.11 \mu \mathrm{M} / \mathrm{L})$ compared to females $(0 \mu \mathrm{M} / \mathrm{L}) ; \mathrm{p}=0.034$. Also, the levels of $\mathrm{C} 16$ were significantly higher in males $(0.81 \mu \mathrm{M} / \mathrm{L})$ than in females $(0 \mu \mathrm{M} / \mathrm{L}) ; \mathrm{p}=0.034$.

Increased age (Table 3 ) positively correlated with increased levels of free carnitine (Spearman's coefficient $0.695 ; \mathrm{p}=0.026)$ and the phenylalanine/tyrosine ratio (Spearman's coefficient 0.818; $\mathrm{p}=0.004$ ) in COPD patients. In the COPD group, a negative correlation with age was found in $\mathrm{C} 5-\mathrm{OH}$ (Spearman's coefficient -0.644; $\mathrm{p}=0.044$ ) and C14:1 (Spearman's coefficient -0.688 ; $\mathrm{p}=0.028$ ). In the control group, age positively correlated with the levels of C14 (Spearman's coefficient 0.765; $\mathrm{p}=0.010$ ) as well as the phenylalanine/tyrosine ratio (Spearman's coefficient 0.694; $\mathrm{p}=0.026$ ) and the phenylalanine level (Spearman's coefficient $0.671 ; \mathrm{p}=0.034)$.

In the COPD group, BMI (Table 4) positively correlated with $\mathrm{C} 0 / \mathrm{AC}$ ratios (Spearman's coefficient 0.758 ; $\mathrm{p}=0.011$, whilst there was a negative correlation with C5/C2 ratios (Spearman's coefficient $-0.809 ; \mathrm{p}=0.005$ ). In the control group, the leucine/isoleucine ratio
Table 3. Influence of age on the results

\begin{tabular}{|c|c|c|c|c|}
\hline \multirow{2}{*}{$\begin{array}{c}\text { Carnitines } \\
\text { and amino } \\
\text { acids }\end{array}$} & \multicolumn{2}{|c|}{$\operatorname{COPD}(n=10)$} & \multicolumn{2}{|c|}{ Control $(n=10)$} \\
\hline & $\begin{array}{l}\text { Spearman's } \\
\text { coefficient }\end{array}$ & $p$-value & $\begin{array}{l}\text { Spearman's } \\
\text { coefficient }\end{array}$ & $p$-value \\
\hline $\mathrm{CO}$ & 0.695 & 0.026 & 0.419 & 0.229 \\
\hline$C 2$ & 0.494 & 0.147 & 0.345 & 0.329 \\
\hline C3 & 0.335 & 0.343 & 0.287 & 0.422 \\
\hline C4 & 0.052 & 0.888 & 0.501 & 0.140 \\
\hline $\mathrm{C} 5$ & -0.017 & 0.962 & -0.069 & 0.850 \\
\hline $\mathrm{C} 5-\mathrm{OH}$ & -0.644 & 0.044 & 0.033 & 0.928 \\
\hline $\mathrm{C} 8$ & -0.157 & 0.664 & -0.174 & 0.630 \\
\hline $\mathrm{C} 14$ & 0.498 & 0.143 & 0.765 & 0.010 \\
\hline C14:1 & -0.688 & 0.028 & 0.182 & 0.615 \\
\hline $\mathrm{C} 16$ & -0.642 & 0.045 & -0.210 & 0.560 \\
\hline $\mathrm{C} 16-\mathrm{OH}$ & -0.328 & 0.355 & -0.092 & 0.800 \\
\hline C18:1 & 0.136 & 0.707 & 0.008 & 0.983 \\
\hline AC total & 0.591 & 0.072 & 0.443 & 0.200 \\
\hline $\mathrm{CO} / \mathrm{AC}$ & 0.134 & 0.712 & 0.086 & 0.813 \\
\hline $\mathrm{C} 3 / \mathrm{C} 16$ & 0.294 & 0.410 & 0.223 & 0.535 \\
\hline $\mathrm{C} 3 / \mathrm{C} 2$ & -0.205 & 0.570 & -0.052 & 0.886 \\
\hline $\mathrm{C} 5 / \mathrm{C} 2$ & -0.224 & 0.534 & -0.118 & 0.745 \\
\hline $\mathrm{C} 8 / \mathrm{C} 2$ & 0.237 & 0.510 & -0.530 & 0.115 \\
\hline C14:1/C16 & -0.202 & 0.575 & 0.004 & 0.990 \\
\hline $\mathrm{C} 16-\mathrm{OH} / \mathrm{C} 16$ & -0.544 & 0.104 & 0.004 & 0.990 \\
\hline Glutaryl & 0.017 & 0.962 & 0.180 & 0.618 \\
\hline Valine & -0.305 & 0.392 & -0.203 & 0.574 \\
\hline Pyg & 0.146 & 0.687 & 0.083 & 0.821 \\
\hline Met & 0.292 & 0.413 & - & - \\
\hline Phe & -0.260 & 0.468 & 0.671 & 0.034 \\
\hline Tyr & 0.250 & 0.486 & 0.364 & 0.301 \\
\hline Cit1 & -0.310 & 0.384 & 0.321 & 0.366 \\
\hline Glyc & -0.280 & 0.432 & 0.499 & 0.142 \\
\hline Alanine & -0.104 & 0.776 & 0.437 & 0.207 \\
\hline Leu/lle & -0.470 & 0.171 & 0.443 & 0.200 \\
\hline Phe/Tyr & 0.818 & 0.004 & 0.694 & 0.026 \\
\hline
\end{tabular}

C0 - free or non-acylated carnitine; C2-C18-carnitine acylated with an acyl containing the indicated number of carbon atoms; - $\mathrm{OH}-$ a hydroxyl group on an acyl; AC - acylcarnitines; Pyg - pyroglutamic acid; Met - methionine; Phe - phenylalanine; Tyr - tyrosine; Cit - citrulline; Glyc - glycine; Leu - leucine; lle - isoleucine.

positively correlated with BMI (Spearman's coefficient $0.721 ; \mathrm{p}=0.019)$.

Fat free mass index had no effect on the metabolomic profile of COPD patients (Table 5). In the COPD group, there was a negative correlation of $\mathrm{FEV}_{1}$ with $\mathrm{C} 3$ (Spearman's coefficient $-0.634 ; \mathrm{p}=0.049$ ), C5 (Spearman's coefficient $-0.676 ; \mathrm{p}=0.032$ ) and leucine/isoleucine ratios (Spearman's coefficient -0.702; $\mathrm{p}=0.024$ ) (Table 6). There was a negative correlation between $\mathrm{FEV}_{1} / \mathrm{FVC}$ and the C4 (Spearman's coefficient $-0.635 ; \mathrm{p}=0.049$ ) and 
tyrosine levels (Spearman's coefficient $-0.770 ; \mathrm{p}=0.009$ ) in the COPD group (Table 7).

\section{Discussion}

The severity of COPD in patients is normally graded based on the degree of obstruction as measured by $\mathrm{FEV}_{1}$. Today, it is accepted that COPD is a complex disease with systemic effects. Transcriptomics, proteomic and metabolomic investigation have the potential to contribute

Table 4. Influence of BMI on the results

\begin{tabular}{|c|c|c|c|c|}
\hline \multirow{2}{*}{$\begin{array}{l}\text { Carnitines and } \\
\text { amino acids }\end{array}$} & \multicolumn{2}{|c|}{$\operatorname{COPD}(n=10)$} & \multicolumn{2}{|c|}{ Control $(n=10)$} \\
\hline & $\begin{array}{l}\text { Spearman's } \\
\text { coefficient }\end{array}$ & $p$-value & $\begin{array}{l}\text { Spearman's } \\
\text { coefficient }\end{array}$ & p-value \\
\hline CO & 0.467 & 0.174 & 0.624 & 0.054 \\
\hline C2 & -0.370 & 0.293 & 0.285 & 0.425 \\
\hline C3 & 0.273 & 0.446 & -0.043 & 0.906 \\
\hline C4 & -0.546 & 0.102 & -0.545 & 0.103 \\
\hline C5 & 0.027 & 0.940 & 0.437 & 0.207 \\
\hline $\mathrm{C} 5-\mathrm{OH}$ & -0.407 & 0.243 & -0.032 & 0.929 \\
\hline C8 & -0.069 & 0.850 & 0.067 & 0.854 \\
\hline C14 & -0.341 & 0.334 & 0.470 & 0.171 \\
\hline C14:1 & -0.294 & 0.409 & -0.231 & 0.521 \\
\hline C16 & -0.362 & 0.304 & 0.252 & 0.482 \\
\hline $\mathrm{C} 16-\mathrm{OH}$ & -0.479 & 0.161 & 0.528 & 0.117 \\
\hline C18:1 & -0.548 & 0.101 & 0.231 & 0.521 \\
\hline AC total & 0.273 & 0.446 & 0.612 & 0.060 \\
\hline CO/AC & 0.758 & 0.011 & 0.455 & 0.187 \\
\hline $\mathrm{C} 3 / \mathrm{C} 16$ & -0.170 & 0.638 & 0.614 & 0.059 \\
\hline $\mathrm{C} 3 / \mathrm{C} 2$ & -0.278 & 0.437 & 0.292 & 0.413 \\
\hline C5/C2 & -0.809 & 0.005 & 0.149 & 0.682 \\
\hline C8/C2 & 0.038 & 0.918 & 0.058 & 0.873 \\
\hline C14:1/C16 & -0.292 & 0.413 & 0.199 & 0.582 \\
\hline $\mathrm{C} 16-\mathrm{OH} / \mathrm{C} 16$ & -0.389 & 0.266 & 0.510 & 0.132 \\
\hline Glutaryl & -0.450 & 0.192 & -0.191 & 0.597 \\
\hline Valine & 0.624 & 0.054 & 0.370 & 0.293 \\
\hline Pyg & 0.394 & 0.260 & 0.194 & 0.592 \\
\hline Met & 0.290 & 0.416 & - & - \\
\hline Phe & -0.498 & 0.143 & 0.612 & 0.060 \\
\hline Tyr & -0.442 & 0.200 & 0.511 & 0.132 \\
\hline Cit1 & -0.006 & 0.987 & 0.389 & 0.266 \\
\hline Glyc & -0.152 & 0.676 & 0.539 & 0.108 \\
\hline Alanine & 0.176 & 0.627 & 0.576 & 0.082 \\
\hline Leu/lle & 0.018 & 0.960 & 0.721 & 0.019 \\
\hline Phe/Tyr & -0.172 & 0.636 & 0.311 & 0.381 \\
\hline
\end{tabular}

C0 - free or non-acylated carnitine; C2-C18 - carnitine acylated with an acyl containing the indicated number of carbon atoms; - OH a hydroxyl group on an acyl; AC - acylcarnitines; Pyg - pyroglutamic acid; Met - methionine; Phe - phenylalanine; Tyr - tyrosine; Cit - citrulline; Glyc - glycine; Leu - leucine; lle - isoleucine. to a better understanding of COPD and its natural course. This is paramount, as COPD has been recently recognized as a condition affecting the whole organism. For example, it was shown that COPD patients are very likely to suffer from metabolic syndrome with increased pro-inflammatory markers from the lungs and adipose tissue. ${ }^{20}$

The development of modern instrumental analytical techniques contributes to the investigation of many pathological states and helps in the elucidation of metabolic and other processes inside the body of a patient. In our work, we investigated the concentrations of various small molecules, mainly various amino acids and free and acylated carnitines with a different length of the alkyl chain, using the HPLCMS/MS method.

Carnitine is a quaternary ammonium compound, synthesized by the kidneys and liver from the amino acids lysine and methionine. It is also obtained through a diet, particularly through red meat and nuts. Carnitine has a major role in the citric acid cycle by binding and transporting various acyl groups to be metabolized through $\beta$-oxidation. Consequently, the disturbance of these processes leads to various pathological states. ${ }^{21,22}$ Genetic disorders, such as primary carnitine deficiency, carnitine palmitoyltransferase deficiency type I and II among others, affect different steps in carnitine biotransformations. ${ }^{23}$ The significantly lower ratio of free carnitine/acylated carnitine (C0/AC) in COPD patients, despite the lack of distinct differences in the concentration of free carnitine, and the total and individual acylcarnitine levels, possibly indicates a predisposition to atherosclerosis as a result of inadequate $\beta$-oxidation of fatty acids, as well as of being at risk for carnitine deficiency. ${ }^{24,25}$

Evidence suggests that impaired fatty acid oxidation has some effect on the development of type-2 diabetes. ${ }^{26,27}$ The prevalence of diabetes in COPD patients is significant, and may be one of the factors which cause the progression and worsen the prognosis of COPD. ${ }^{28-30}$ Acylcarnitines have a potential to activate proinflammatory signaling pathways as part of the shared mechanism of COPD and cardiovascular disease. ${ }^{27,31}$ Metabolic heritability may be implicated in aberrant levels of some acylcarnitines, as it was shown that some metabolite levels are inheritable at birth and that some genes are associated with acylcarnitines. ${ }^{32}$

The role of inherited fatty acid oxidation deficiency and genetics in COPD may be further implicated by the higher levels of the acylated $\mathrm{C} 8 / \mathrm{C} 2$ carnitines ratio in COPD patients. The $\mathrm{C} 8 / \mathrm{C} 2$ ratio and the $\mathrm{C} 8$ levels are the most accurate markers of medium-chain acyl-CoA dehydrogenase deficiency (MCADD), the most common inherited defect in the fatty acid oxidation pathway marked by an inability to break down medium-chain fatty acids during periods of fasting. ${ }^{33}$

Additional findings relate to alanine, a nonessential amino acid found in a variety of foods. It may be synthesized by the body itself from pyruvate and a branched-chain 
Table 5. Influence of FFMI on the results

\begin{tabular}{|c|c|c|}
\hline \multirow{2}{*}{$\begin{array}{c}\text { Carnitines and } \\
\text { amino acids }\end{array}$} & \multicolumn{2}{|c|}{$\operatorname{COPD}(n=10)$} \\
\hline & $\begin{array}{l}\text { Spearman's } \\
\text { coefficient }\end{array}$ & $p$-value \\
\hline $\mathrm{CO}$ & 0.176 & 0.627 \\
\hline $\mathrm{C} 2$ & -0.103 & 0.777 \\
\hline C3 & 0.030 & 0.934 \\
\hline C4 & -0.171 & 0.637 \\
\hline C5 & -0.191 & 0.597 \\
\hline $\mathrm{C} 5-\mathrm{OH}$ & -0.226 & 0.530 \\
\hline $\mathrm{C} 8$ & -0.144 & 0.692 \\
\hline $\mathrm{C} 14$ & -0.178 & 0.624 \\
\hline C14:1 & -0.156 & 0.668 \\
\hline $\mathrm{C} 16$ & 0.018 & 0.960 \\
\hline $\mathrm{C} 16-\mathrm{OH}$ & -0.045 & 0.902 \\
\hline C18:1 & -0.363 & 0.302 \\
\hline AC total & 0.188 & 0.603 \\
\hline CO/AC & 0.212 & 0.556 \\
\hline $\mathrm{C} 3 / \mathrm{C} 16$ & -0.055 & 0.881 \\
\hline $\mathrm{C} 3 / \mathrm{C2}$ & 0.222 & 0.537 \\
\hline $\mathrm{C} 5 / \mathrm{C} 2$ & -0.507 & 0.135 \\
\hline $\mathrm{C} 8 / \mathrm{C} 2$ & -0.063 & 0.863 \\
\hline C14:1/C16 & 0.019 & 0.957 \\
\hline $\mathrm{C} 16-\mathrm{OH} / \mathrm{C} 16$ & 0.130 & 0.721 \\
\hline Glutaryl & -0.623 & 0.054 \\
\hline Valine & 0.661 & 0.038 \\
\hline Pyg & 0.624 & 0.054 \\
\hline Met & 0.058 & 0.873 \\
\hline Phe & -0.140 & 0.700 \\
\hline Tyr & -0.539 & 0.108 \\
\hline Cit1 & 0.299 & 0.402 \\
\hline Glyc & 0.006 & 0.987 \\
\hline Alanine & 0.430 & 0.214 \\
\hline Leu/lle & 0.152 & 0.676 \\
\hline Phe/Tyr & -0.455 & 0.187 \\
\hline
\end{tabular}

CO - free or non-acylated carnitine; C2-C18 - carnitine acylated with an acyl containing the indicated number of carbon atoms; - $\mathrm{OH}$ - a hydroxyl group on an acyl; AC - acylcarnitines; Pyg - pyroglutamic acid; Met - methionine; Phe - phenylalanine Tyr - tyrosine; Cit - citrulline; Glyc - glycine; Leu - leucine; lle - isoleucine.
Table 6. Influence of FEV ${ }_{1}$ on the results

\begin{tabular}{|c|c|c|}
\hline \multirow{2}{*}{$\begin{array}{l}\text { Carnitines and } \\
\text { amino acids }\end{array}$} & \multicolumn{2}{|c|}{$\operatorname{COPD}(n=10)$} \\
\hline & $\begin{array}{l}\text { Spearman's } \\
\text { coefficient }\end{array}$ & p-value \\
\hline $\mathrm{CO}$ & -0.098 & 0.787 \\
\hline $\mathrm{C} 2$ & -0.055 & 0.879 \\
\hline $\mathrm{C} 3$ & -0.634 & 0.049 \\
\hline C4 & -0.170 & 0.639 \\
\hline $\mathrm{C} 5$ & -0.676 & 0.032 \\
\hline $\mathrm{C} 5-\mathrm{OH}$ & 0.158 & 0.664 \\
\hline $\mathrm{C} 8$ & -0.410 & 0.240 \\
\hline $\mathrm{C} 14$ & 0.132 & 0.717 \\
\hline C14:1 & 0.439 & 0.204 \\
\hline C16 & 0.305 & 0.391 \\
\hline $\mathrm{C} 16-\mathrm{OH}$ & -0.053 & 0.884 \\
\hline C18:1 & 0.194 & 0.592 \\
\hline AC total & -0.154 & 0.671 \\
\hline CO/AC & 0.037 & 0.919 \\
\hline C3/C16 & 0.120 & 0.740 \\
\hline $\mathrm{C} 3 / \mathrm{C} 2$ & 0.392 & 0.263 \\
\hline $\mathrm{C} 5 / \mathrm{C} 2$ & 0.097 & 0.789 \\
\hline $\mathrm{C} 8 / \mathrm{C} 2$ & -0.628 & 0.052 \\
\hline $\mathrm{C} 14: 1 / \mathrm{C} 16$ & 0.573 & 0.083 \\
\hline $\mathrm{C} 16-\mathrm{OH} / \mathrm{C} 16$ & -0.141 & 0.699 \\
\hline Glutaryl & 0.044 & 0.904 \\
\hline Valine & -0.406 & 0.244 \\
\hline Pyg & 0.579 & 0.080 \\
\hline Met & 0.530 & 0.115 \\
\hline Phe & -0.096 & 0.793 \\
\hline Tyr & -0.474 & 0.166 \\
\hline Cit1 & -0.180 & 0.620 \\
\hline Glyc & -0.031 & 0.933 \\
\hline Alanine & -0.369 & 0.294 \\
\hline Leu/Ile & -0.702 & 0.024 \\
\hline Phe/Tyr & 0.083 & 0.819 \\
\hline
\end{tabular}

CO - free or non-acylated carnitine;

C2-C18 - carnitine acylated with an acyl containing the indicated number of carbon atoms; -OH - a hydroxyl group on an acyl;

AC - acylcarnitines; Pyg - pyroglutamic acid; Met - methionine; Phe - phenylalanine;

Tyr - tyrosine; Cit - citrulline; Glyc - glycine; Leu - leucine; lle - isoleucine.
Table 7. Influence of FEV $1 / F V C$ on the results

\begin{tabular}{|c|c|c|}
\hline \multirow{2}{*}{$\begin{array}{l}\text { Carnitines and } \\
\text { amino acids }\end{array}$} & \multicolumn{2}{|c|}{$\operatorname{COPD}(n=10)$} \\
\hline & $\begin{array}{l}\text { Spearman's } \\
\text { coefficient }\end{array}$ & $p$-value \\
\hline $\mathrm{CO}$ & 0.248 & 0.489 \\
\hline $\mathrm{C} 2$ & -0.285 & 0.425 \\
\hline C3 & -0.248 & 0.489 \\
\hline C4 & -0.635 & 0.049 \\
\hline C5 & -0.478 & 0.162 \\
\hline $\mathrm{C} 5-\mathrm{OH}$ & 0.175 & 0.630 \\
\hline $\mathrm{C} 8$ & -0.344 & 0.331 \\
\hline $\mathrm{C} 14$ & -0.417 & 0.231 \\
\hline C14:1 & 0.372 & 0.290 \\
\hline $\mathrm{C} 16$ & 0.288 & 0.419 \\
\hline $\mathrm{C} 16-\mathrm{OH}$ & -0.539 & 0.108 \\
\hline C18:1 & -0.006 & 0.987 \\
\hline AC total & 0.067 & 0.855 \\
\hline CO/AC & 0.358 & 0.310 \\
\hline $\mathrm{C} 3 / \mathrm{C} 16$ & -0.261 & 0.466 \\
\hline $\mathrm{C} 3 / \mathrm{C} 2$ & -0.198 & 0.584 \\
\hline $\mathrm{C} 5 / \mathrm{C} 2$ & -0.343 & 0.332 \\
\hline $\mathrm{C} 8 / \mathrm{C} 2$ & -0.496 & 0.145 \\
\hline C14:1/C16 & -0.091 & 0.803 \\
\hline $\mathrm{C} 16-\mathrm{OH} / \mathrm{C} 16$ & -0.389 & 0.266 \\
\hline Glutaryl & 0.294 & 0.409 \\
\hline Valine & 0.127 & 0.726 \\
\hline Pyg & 0.370 & 0.293 \\
\hline Met & 0.174 & 0.631 \\
\hline Phe & 0.030 & 0.934 \\
\hline Tyr & -0.770 & 0.009 \\
\hline Cit1 & -0.439 & 0.204 \\
\hline Glyc & 0.176 & 0.627 \\
\hline Alanine & -0.406 & 0.244 \\
\hline Leu/lle & -0.103 & 0.777 \\
\hline Phe/Tyr & -0.216 & 0.548 \\
\hline
\end{tabular}

CO - free or non-acylated carnitine;

C2-C18 - carnitine acylated with an acyl containing the indicated number of carbon atoms; -OH - a hydroxyl group on an acyl; AC - acylcarnitines; Pyg - pyroglutamic acid; Met - methionine; Phe - phenylalanine; Tyr - tyrosine; Cit - citrulline; Glyc - glycine; Leu - leucine; lle - isoleucine. amino acid. Dietary sources include meat, seafood, dairy, eggs, beans, and nuts. It is an important source of muscle energy. Alanine is an amino acid linked to energy metabolismrelated pathways of glycolysis, gluconeogenesis, the alanine cycle, and the citric acid cycle. ${ }^{34}$ Decreased levels of alanine were reported earlier in relation to COPD and muscle wasting because of disturbed metabolism of proteins. ${ }^{35}$ Our finding of decreased levels of alanine in patients with COPD compared to the control group may indicate a risk of protein malnutrition as do significantly lower levels of phenylalanine in COPD patients compared to the control group.

Higher levels of pyroglutamate in COPD patients are of unknown significance and should be researched further.

These are important findings, as natural physiological concentrations of substrates related to energy metabolism (alanine, phenylalanine and carnitines), and consequently to malnutrition, are disturbed in COPD patients, while the concentrations of substrates not linked directly 
to energy metabolism (other amino acids) are not significantly compromised.

Chronic malnutrition is a common problem in a myriad of chronic diseases, COPD and heart disease being amongst them. ${ }^{36,37}$ It is connected to complications during hospitalizations, a higher rate of readmissions, increased costs of care, and the loss of quality of life. ${ }^{36,38}$ Malnutrition in COPD is a negative predictive factor, being strongly associated with in-hospital mortality and readmittance 30 days after discharge. ${ }^{39,40}$ Skeletal muscles are affected by inactivity, unbalanced nutrition, age, and inflammation, leading to sarcopenia, which involves both muscle loss and muscle dysfunction with contractile impairment and metabolic anomalies. These responses are poorly understood (and are beyond the scope of this article), but 2 groups of patients may be identified: cachectic and with normal or increased BMI. Malnutrition may be present in cachectic patients as well as in patients with normal or increased BMI, i.e., sarcopenia and sarcopenic obesity. ${ }^{41}$ Nutritional intervention is beneficial for malnourished patients, combined with exercise or a rehabilitation program, which, in the long term, may be associated with lower health care costs. ${ }^{36,38,42}$ This is already being echoed by some national guidelines. ${ }^{2}$

Metabolomic profiling using the HPLC-MS/MS method is a new application in the investigation of COPD, and as such it serves as a source of essential information on processes that occur in COPD patients' bodies. Our results were obtained in the limited number of patients. We intend to continue our research in this direction, as it is highly desirable that future studies involve a higher number of subjects to investigate a varying role of individual comorbidities and physiological COPD parameters in the long term to elucidate further COPD pathology.

\section{Limitations}

This was a pilot study conducted on a small number of patients. The control group was younger than the COPD test subjects, although not statistically significantly. This was due to the strict exclusion criteria, namely smokers or exsmokers of less than 6 months, and the presence of metabolic diseases including diabetes mellitus types 1 and 2 . There is a high prevalence of diabetes mellitus in the Czech Republic, which is $8 \%$, compared to the estimated worldwide prevalence of $2.8-4.4 \%{ }^{43,44}$ As the incidence of diabetes mellitus increases with age, the set criteria excluded most of our older control group candidates from the study. ${ }^{44}$ Further studies on significantly larger cohorts are needed.

Patients with chronic obstructive pulmonary disease are commonly afflicted with comorbidities, particularly coronary artery disease. As there is no data available on the metabolomic effects of cardiovascular diseases on the metabolism of COPD patients, future investigation on whether a cardiovascular disease concomitant with
COPD further contributes to metabolomic changes may be beneficial.

As this pilot study already shows, there are metabolic differences between COPD patients and the control group, which makes future investigations of this kind rational.

\section{Conclusions}

Our study shows that the HPLC-MS/MS method is able to show minute changes in the metabolism of patients shown on a molecular level. The significance of many of these differences is not yet understood. The carnitine and acylcarnitine levels in COPD subjects in this study possibly indicate a predisposition to atherosclerosis as a result of inadequate $\beta$-oxidation of fatty acids and show the presence of oxidative stress. Furthermore, the high sensitivity to changes in circulating amino acid levels may allow us to detect subclinical malnutrition and take early preventative interventions such as nutritional supplementation and patient education.

\section{References}

1. Global Initiative for Chronic Obstructive Lung Disease (GOLD) Global strategy for the diagnosis, management, and prevention of chronic obstructive pulmonary disease. http://www.goldcopd. org/uploads/users/files/GOLD_Report_2014_Jun11.pdf. Accessed on March 3, 2015.

2. Koblizek V, Chlumsky J, Zindr V, et al. Chronic obstructive pulmonary disease: Official diagnosis and treatment guidelines of the Czech Pneumological and Phthisiological Society; A novel phenotypic approach to COPD with patient-oriented care. Biomed Pap Med Fac Univ Palacky Olomouc Czech Repub. 2013;157:189-201.

3. Suzuki T, Tada Y, Kawata N, et al. Clinical, physiological, and radiological features of asthma-chronic obstructive pulmonary disease overlap syndrome. Int J Chron Obstruct Pulmon Dis. 2015;10:947-954.

4. Laviolette L, Laveneziana P; ERS Research Seminar Faculty. Dyspnoea: A multidimensional and multidisciplinary approach. Eur Respir J. 2014;43:1750-1762.

5. Waschki B, Kirsten AM, Holz O, et al. Disease progression and changes in physical activity in patients with COPD. Am J Respir Crit Care Med. 2015;192:295-306.

6. Gika HG, Wilson ID, Theodoridis GA. LC-MS-based holistic metabolic profiling. Problems, limitations, advantages, and future perspectives. J Chromatogr B Analyt Technol Biomed Life Sci. 2014;966:1-6.

7. Gika HG, Theodoridis GA, Plumb RS, Wilson ID. Current practice of liquid chromatography-mass spectrometry in metabolomics and metabonomics. J Pharm Biomed Anal. 2014;87:12-25.

8. Basanta M, Jarvis RM, Xu Y, et al. Non-invasive metabolomic analysis of breath using differential mobility spectrometry in patients with chronic obstructive pulmonary disease and healthy smokers. Analyst. 2010;135:315-320.

9. Fens N, de Nijs SB, Peters S, et al. Exhaled air molecular profiling in relation to inflammatory subtype and activity in COPD. Eur Respir J. 2011;38:1301-1309.

10. de Laurentiis G, Paris D, Melck D, et al. Separating smoking-related diseases using NMR-based metabolomics of exhaled breath condensate. J Proteome Res. 2013;12:1502-1511.

11. de Laurentiis G, Paris D, Melck D, et al. Metabonomic analysis of exhaled breath condensate in adults by nuclear magnetic resonance spectroscopy. Eur Respir J. 2008;32:1175-1183.

12. Wang L, Tang Y, Liu S, et al. Metabonomic profiling of serum and urine by (1)H NMR-based spectroscopy discriminates patients with chronic obstructive pulmonary disease and healthy individuals. PLoS One. 2013;8:e65675. 
13. McClay JL, Adkins DE, Isern NG, et al. (1)H nuclear magnetic resonance metabolomics analysis identifies novel urinary biomarkers for lung function. J Proteome Res. 2010;9:3083-3090.

14. Ubhi BK, Riley JH, Shaw PA, et al. Metabolic profiling detects biomarkers of protein degradation in COPD patients. Eur Respir J. 2012;40:345-355.

15. Ubhi BK, Cheng KK, Dong J, et al. Targeted metabolomics identifies perturbations in amino acid metabolism that sub-classify patients with COPD. Mol Biosyst. 2012;8:3125-3133.

16. Paige M, Burdick MD, Kim S, Xu J, Lee JK, Michael Shim Y. Pilot analysis of the plasma metabolite profiles associated with emphysematous chronic obstructive pulmonary disease phenotype. Biochem Biophys Res Commun. 2011;413:588-593.

17. Fumagalli $M$, Ferrari $F$, Luisetti $M$, et al. Profiling the proteome of exhaled breath condensate in healthy smokers and COPD patients by LC-MS/MS. Int J Mol Sci. 2012;13:13894-13910.

18. Tu C, Mammen MJ, Li J, et al. Large-scale, ion-current-based proteomics investigation of bronchoalveolar lavage fluid in chronic obstructive pulmonary disease patients. J Proteome Res. 2014;13:627-639.

19. Merali S, Barrero CA, Bowler RP, et al. Analysis of the plasma proteome in COPD: Novel low abundance proteins reflect the severity of lung remodeling. COPD. 2014;11:177-189.

20. Clini E, Crisafulli E, Radaeli A, Malerba M. COPD and the metabolic syndrome: An intriguing association. Intern Emerg Med. 2013;8:283-289.

21. Schooneman MG, Vaz FM, Houten SM, Soeters MR. Acylcarnitines: Reflecting or inflicting insulin resistance? Diabetes. 2013;62:1-8.

22. Patel SG, Hsu JW, Jahoor F, et al. Pathogenesis of $A^{-} \beta^{+}$ketosis-prone diabetes. Diabetes. 2013;62:912-922.

23. Lin X, Shim K, Odle J. Carnitine palmitoyltransferase I control of acetogenesis, the major pathway of fatty acid \{beta\}-oxidation in liver of neonatal swine. Am J Physiol Regul IntegrComp Physiol.2010;298:R1435-1443.

24. Blair HC, Sepulveda J, Papachristou DJ. Nature and nurture in atherosclerosis: The roles of acylcarnitine and cell membrane-fatty acid intermediates. Vascul Pharmacol. June 30, 2015. doi:10.1016/j. vph.2015.06.012

25. Rauschert S, Uhl O, Koletzko B, Hellmuth C. Metabolomic biomarkers for obesity in humans: A short review. Ann Nutr Metab. 2014;64:314-324.

26. Anderson SG, Dunn WB, Banerjee $M$, et al. Evidence that multiple defects in lipid regulation occur before hyperglycemia during the prodrome of type-2 diabetes. PLoS One. 2014;9:e103217.

27. Rutkowsky JM, Knotts TA, Ono-Moore KD, et al. Acylcarnitines activate proinflammatory signaling pathways. Am J Physiol Endocrinol Metab. 2014;306:E1378-1387.

28. Hersh CP, Make BJ, Lynch DA, et al. Non-emphysematous chronic obstructive pulmonary disease is associated with diabetes mellitus. BMC Pulm Med. 2014;14:164.

29. Rogliani P, Calzetta L, Segreti A, Barrile A, Cazzola M. Diabetes mellitus among outpatients with COPD attending a university hospital. Acta Diabetol. 2014;51:933-940.

30. Gläser S, Krüger S, Merkel M, Bramlage P, Herth FJ. Chronic obstruc tive pulmonary disease and diabetes mellitus: A systematic review of the literature. Respiration. 2015;89:253-264.

31. Vanfleteren LE, Spruit MA, Groenen M, et al. Clusters of comorbidities based on validated objective measurements and systemic inflammation in patients with chronic obstructive pulmonary disease. Am J Respir Crit Care Med. 2013;187:728-735.

32. Ryckman KK, Smith CJ, Jelliffe-Pawlowski LL, Momany AM, Berberich SL, Murray JC. Metabolic heritability at birth: Implications for chronic disease research. Hum Genet. 2014;133:1049-1057.

33. Couce ML, Sánchez-Pintos $P$, Diogo L, et al. Newborn screening for medium-chain acyl-CoA dehydrogenase deficiency: Regional experience and high incidence of carnitine deficiency. Orphanet J Rare Dis. 2013;8:102.

34. Nelson DL, Cox MM. Principles of Biochemistry. $4^{\text {th }}$ ed. New York, NY: W. H. Freeman; 2005:684-685.

35. Pouw EM, Schols AM, Deutz NE, Wouters EF. Plasma and muscle amino acid levels in relation to resting energy expenditure and inflammation in stable chronic obstructive pulmonary disease. Am J Respir Crit Care Med. 1998;158:797-801.

36. Schols AM, Ferreira IM, Franssen FM, et al. Nutritional assessment and therapy in COPD: A European Respiratory Society statement. Eur Respir J. 2014;44:1504-1520.
37. Tevik K, Thürmer H, Husby MI, de Soysa AK, Helvik AS. Nutritional risk screening in hospitalized patients with heart failure. Clin Nutr. 2015;34:257-264.

38. Steiber A, Hegazi R, Herrera M, et al. Spotlight on global malnutrition: A continuing challenge in the $21^{\text {st }}$ century. J Acad Nutr Diet. 2015;115:1335-1341.

39. Zapatero A, Barba R, Ruiz J, et al. Malnutrition and obesity: Influence in mortality and readmissions in chronic obstructive pulmonary disease patients. J Hum Nutr Diet. 2013;26(Suppl 1):16-22.

40. Hanson C, Rutten EP, Wouters EF, Rennard S. Influence of diet and obesity on COPD development and outcomes. Int J Chron Obstruct Pulmon Dis. 2014;9:723-733.

41. Biolo G, Cederholm T, Muscaritoli M. Muscle contractile and metabolic dysfunction is a common feature of sarcopenia of aging and chronic diseases: From sarcopenic obesity to cachexia. Clin Nutr. 2014;33:737-748.

42. Schols AM. Pulmonary cachexia. Int J Cardiol. 2002;85:101-110.

43. Brož J, Brabec M, Žd'árská DJ, Novotná M, Kvapil M. Incidence of diabetes mellitus narrowly correlates with unemployment rate during 2000-2012 in the Czech Republic. Cent Eur J Public Health. 2016;24(1):86-87. doi:10.21101/cejph.a4680

44. Wild S, Roglic G, Green A, Sicree R, King H. Global prevalence of diabetes: Estimates for the year 2000 and projections for 2030. Diabetes Care. 2004;27(5):1047-1053. 